\title{
No Association Between Types of Unilateral Mandibular Condylar Abnormalities and Facial Asymmetry in orthopedic treated Patients with Juvenile Idiopathic Arthritis
}

\section{Highlights}

- Dentofacial asymmetries often occurred in patients with juvenile idiopathic arthritis (JIA).

- Dentofacial development was affected more severely in patients with condylar abnormalities.

- Dysmorphic dentofacial development was not associated with a specific type of condylar abnormality.

- Combined condylar deformations and erosions were observed in one third of the TMJs in JIA. 


\begin{abstract}
Introduction: Dentofacial asymmetries are often observed in patients with juvenile idiopathic arthritis (JIA) and temporomandibular joint (TMJ) involvements. The aim of this split-face study was to associate types of radiological TMJ abnormalities with the degree of dentofacial asymmetry in patients with unilateral TMJ involvements assessed with cone beam computerized tomography (CBCT).

Methods: Forty-seven JIA patients and 19 non-arthritic control subjects were included. Normal condylar radiological CBCT appearance in a minimum of one TMJ was set as the inclusion criterion for all patients with JIA. The contralateral TMJ was thereafter scored as either "normal", "deformed" or "erosive" consistent with predefined criteria. Based on the bilateral radiological TMJ appearances, three JIA groups were assigned: 1) normal/normal, 2) normal/deformed, and 3) normal/erosive. The severity of dentofacial asymmetry was compared between the JIA groups and control subjects. Dentofacial asymmetry was expressed as interside ratios and angular measurements.

Results: Eighty-seven percent of the JIA patients were currently being treated or had previously received treatment with functional orthopedic appliance at the time of the CBCT. Significantly greater dentofacial asymmetries were observed in the two groups of JIA patients with unilateral condylar abnormalities (deformation or erosion) than in the other groups. A similar degree of dentofacial asymmetry was observed in JIA patients with bilateral normal TMJs and in the nonarthritic control group.

Conclusion: JIA patients with unilateral condylar abnormalities (deformation or erosion) exhibited significantly more severe dentofacial asymmetries than JIA patients without condylar abnormalities and control subjects. We found the same degree of dentofacial asymmetry when dividing condylar abnormalities into deformation or erosion groups.
\end{abstract}




\section{Introduction}

The involvement of the temporomandibular joint (TMJ) is a common finding in patients with juvenile idiopathic arthritis (JIA) and has a varying prevalence of up to $96 \%$. ${ }^{1}$ A clinical consequence of TMJ involvement can be dysmorphic mandibular development resulting in dentofacial asymmetry, occlusal instability, alterations in muscular activity, and suboptimal TMJ function, which, in turn, may lead to the development of orofacial symptoms and a compromised aesthetic appearance. ${ }^{2-9}$ One of the primary treatment goals in JIA is to avoid this unwanted dysmorphic dentofacial development. However, the underlying processes are not yet fully understood.

Historically, dysmorphic dentofacial alterations in JIA patients have been regarded as a consequence of arthritis-induced TMJ degeneration resulting in the loss of condylar vertical height, which is an arthritis-induced degradation similar to what occurs in adult patients with rheumatoid arthritis with TMJ involvement. ${ }^{10}$ For decades, extensive research has fueled debates on the implications of TMJ lesions/degeneration on mandibular development and growth. ${ }^{11-14}$ However, the contemporary dominant theory explains the dysmorphic dentofacial development in JIA patients as a consequence of condylar growth disturbances rather than arthritis-induced condylar damage alone. ${ }^{9,15,16-18}$ In a recent review, Peltomäki et al. supported this perception and emphasized the need for documentation in future prospective longitudinal studies. ${ }^{19}$ They further noted that altered dentofacial development may be caused by a combination of inflammatory effects on the intra-articular growth site of the condylar cartilage and impaired masticatory function. ${ }^{19}$

There is currently a need to elucidate the intra-articular processes that occur during dysmorphic dentofacial development in JIA. This is important from a pathogenic perspective to advance our diagnostic and therapeutic understanding of this challenging condition. Combined imaging/radiological TMJ scoring systems have recently been published exclusively for JIA. ${ }^{20,21}$ Components of these scoring systems aim to score the severity of arthritis-induced osseous changes. The scoring systems imply the progression of the arthritis-induced condylar osseous changes from minor condylar head deformation (flattening) to condylar destructions that progressively reduce normal mandibular growth. ${ }^{20,21}$ Both scoring systems grade erosive osseous changes higher than condylar deformation (flattening). With an isolated focus on condylar conditions, these scoring systems may be useful for radiological TMJ assessment. However, no human studies have determined if particular types of abnormal TMJ condylar 
osseous changes (e.g., erosive changes) are associated with a greater extent of mandibular dysmorphic development than others (e.g., condylar deformations). Notably, previous experimental studies found no relationship between the severity of condylar lesions and a reduction in mandibular growth and development in young growing rabbits with experimentally induced and histologically confirmed TMJ arthritis. 17,22

Previous research on TMJ abnormality and dentofacial development in JIA was primarily based on conventional radiological techniques, ${ }^{11,14,23,24}$ and only few studies with more advanced techniques exist. ${ }^{25-29}$ The introduction of cone beam computed tomography (CBCT), a cost- and dose-effective three-dimensional imaging modality, has enabled the radiological examination of TMJ hard tissue pathologies, which is superior to conventional radiological methods. ${ }^{30}$ The aim of this cross-sectional CBCT-based study was to associate radiological TMJ abnormalities with the degree of dentofacial asymmetry in patients with unilateral TMJ involvements. A control group was used to compare asymmetries to the normal population.

\section{Materials and Methods}

\section{JIA patients}

Eighty-six consecutive JIA patients at the Section of Orthodontics; Aarhus University, Denmark were eligible for inclusion. All candidates had received a craniofacial CBCT scan between February 2011 and April 2014. Patients were included when complying with the following inclusion criteria: 1) a diagnosis of JIA according to the ILAR criteria ${ }^{31}$ and 2) at least one TMJ with radiological normal/healthy osseous TMJ appearance. The exclusion criteria were 1) patients with previous craniofacial traumas or patients with an underlying diagnosis of a syndrome or congenital birth defect involving the craniofacial area or 2) CBCT images of poor quality. The data on patient characteristics were collected from medical hospital records. The present study was approved by the Danish Health and Medicines Authorities (DOK2129859) and the Danish Data Protection Agency (2007-58-0010) and was conducted in accordance with the ethics of the Helsinki Declaration.

\section{Control group}

Nineteen controls without JIA previously treated at the Section of Orthodontics, Aarhus University, Denmark, for other reasons were identified. All had received a full-face CBCT scan in relation to an orthodontic treatment. Control subject criteria were: 1) no previous or current diagnosis of temporomandibular dysfunction; 2) a high-quality CBCT allowing optimal 
radiological assessment; and 3) an age comparable to the JIA patients. Although control subjects with a neutral dental occlusion (Class I) were preferred, full-face CBCTs were rarely taken in this group; we therefore accepted subjects with dental class II subdivision malocclusions and impacted canines.

\section{CBCT scan}

CBCT scans (NewTom 5G, QR s.r.l., Italy) were acquired in accordance with the manufacturer's instructions. The image acquisition parameters included a FOV of $18 \times 16 \mathrm{~cm}$, a scanning time of approximately $18 \mathrm{sec}$, and $3.6 \mathrm{sec}$ of active radiation (pulsed mode) with settings of $110 \mathrm{kV}$ and 3-7 mA. All CBCT scans were reconstructed with a 0.30-mm isotropic voxel dimension.

\section{Radiological TMJ evaluation}

The radiological evaluation was carried out on the joint level, giving each individual two independently assessed TMJ scores (one score for the right joint and one score for the left joint). The interpretation of the CBCT images was conducted using NewTom NNT Viewer software (version 4.6). The type of mandibular condylar abnormality was independently scored by a specially trained maxillofacial radiologist (LZA) who was blinded to the patient diagnosis and the order in which the TMJs were presented (control and JIA subjects were mixed). Cropped CBCT images only including the TMJs were viewed in axial, oblique coronal and oblique sagittal planes. The radiologist was therefore also blinded to the presence of any dentofacial asymmetry. Prior to patient inclusion, three definitions of condylar scores were decided based on a categorization of the condylar radiological appearance (Fig. 1):

1. Normal: normal shape with smooth and intact outline/surface (score 0 )

2. Deformed: marked flattening or other changes in shape with smooth and intact outline/surface (score A)

3. Erosive: disruption of outline or uneven surface due to cysts or erosion (score B)

The maxillofacial radiologist was asked to subgroup each mandibular condyle based on the radiological appearances into one of three groups: normal condylar outline (score 0) or abnormal condylar outline (score A or B). When both deformations (score A) and erosive changes (score B) were found in the same TMJ, the score was based on the most prominent 
radiological feature present. Each individual was thereafter categorized by the bilateral joint scores and assigned to one of six groups: JIA 0-0, JIA 0-A, JIA 0-B, JIA A-A, JIA A-B or JIA B-B. In compliance with the inclusion criteria of this split-face designed study, only patients from the JIA 0-0, JIA 0-A, and JIA 0-B sub-groups were included in our data analyses because a minimum of one joint in each patient was required to have a normal appearance. The control subjects were also sub-grouped based on their bilateral joint scores. However, to represent the population-based variation in radiological condylar appearance, all control subjects were accepted without considering their bilateral joint scores. Therefore, control subjects with radiological abnormalities A-A or B-B were also included. Double assessments of 20 consecutive TMJs were conducted with a two-week interval to assess the intra-observer agreement.

\section{$3 D$ analysis}

To assess the degree of dentofacial asymmetry, a three-dimensional dentofacial analysis was conducted using Mimics software 16.0 (Materialise Interactive Medical Image Control System, Leuven, Belgium). The aim was to assess the intra-patient mandibular asymmetries based on outcome variables representing facial asymmetries between the left and right side of the face. Information on sagittal, vertical, and transversal mandibular asymmetries was collected using eight anatomical landmarks. The landmarks are defined and presented in Table 1 and Fig. 2.

\section{Reference planes}

For the analysis, two main reference planes were constructed:

1. Axial plane: through S and LOr and LOl.

2. Midsagittal plane: through $\mathrm{N}, \mathrm{S}$ and perpendicular to the axial plane.

The additional planes necessary for the analysis were constructed with reference to these two planes. The points and additional planes are defined in Table 1 and shown in Fig. 2 ab.

\section{Outcome variables of craniofacial asymmetry}

A total of nine predefined outcome variables were assessed (Table 1, Fig. 2cd). In the vertical direction, five bilateral length variables of interest were defined: 1) total mandibular height (from Co to Go), 2) condylar height (from Co to Inc plane), 3) ramus height (from Go to Inc plane), 4) the distance from the axial plane to the gonion, and 5) the distance from the axial 
plane to Minf. One variable was defined in the sagittal direction; the length of the mandible left and right side (from Go to $\mathrm{Gn}$ ). In the transversal direction, one variable was defined: transversally, the distance from the midsagittal plane to the left and right gonion. Additionally, two angular measurements were defined: 1) the jaw angle left and right side (the angle between the line connecting $\mathrm{Co}$ and Go and the line connecting Go and Gn) and 2) the gonial plane angle (the angle formed between a plane passing through the right gonion and the left gonion (perpendicular to the coronal plane) and the midsagittal plane (degrees) measured to the affected side). In group 0-0, the angle was measured to the shortest side. For all bilateral outcome variables, a ratio of asymmetry was calculated to obtain a measure of intra-patient dentofacial asymmetry. The ratio was calculated by dividing the value of the affected side (TMJ score A or B) with the value of the non-affected side (TMJ score 0). In group 0-0, the ratio was calculated using the smallest value divided by the largest value.

\section{Statistics}

The data concerning the nine outcome variables were tested for a normal distribution by visual inspections of Q-Q plots. Inter-group differences in dentofacial asymmetry were assessed using analysis of variance (ANOVA) tests with independent Student's $t$-tests serving as postANOVA tests. Post-ANOVA testing was only performed in outcome variables where a statistically significant difference was observed in the primary ANOVA test. The significance level was adjusted in accordance with Bonferroni to avoid a type 1 error due to multiple testing. In the primary ANOVA tests, the significance level was lowered with the number of tests conducted $(0.05 / 9 \rightarrow p<0.006)$. In the post-ANOVA tests, the significance level was adjusted based on the number of $t$-tests conducted within each individual outcome variable $(0.05 / 6 \rightarrow$ $\mathrm{p}<0.008)$.

Intra-rater agreement of the radiological condylar TMJ scores was assessed by kappa statistics based on duplicate assessments two weeks apart. The error of the method of the nine outcome variables describing craniofacial asymmetry was also evaluated based on duplicate assessments two weeks apart using scatterplots and correlation coefficients. ${ }^{32}$ For each of the nine outcome variables, the error of measurement was assessed based on Bland-Altman plots. ${ }^{32}$ The limits of agreements derived from the Bland-Altman plots were used to define the smallest detectable difference for each specific outcome variable: The smallest detectable difference was defined as the minimal amount of change in the inter-side ratios and angles needed to overcome the measurement error within each of the specific outcome variables defined. 


\section{Results}

JIA groups: Forty-seven of the examined 86 patients met the inclusion criteria. All patients presented inflammatory involvements in other joints besides the TMJs. Due to similar bilaterally abnormal radiological TMJ assessment scores (JIA A-A, JIA B-B and JIA A-B), 36 patients $(41 \%)$ were excluded from the study. Three JIA patients were excluded due to low CBCT quality (Fig. 3). The remaining 47 included JIA patients who were grouped into three sub-groups based on the radiological findings: JIA 0-0 $(n=17)$, JIA 0-A $(n=20)$, and JIA 0-B $(n=10)$. The mean ages of the patients in the sub-groups were 13.2 years (std. 2.6) in the JIA 0-0 group, 12.8 years (std. 2.7) in the JIA 0-A group and 11.4 years (std. 2.5) in the JIA 0-B group (Table 2). Comparable distribution of disease duration of TMJ arthritis was observed among the JIA groups (Table 2). Nine of the 47 patients (19\%) had radiological findings of both score A and B within the same joint. Forty-one of the included 47 JIA patients were currently being treated or had previously been treated with a functional orthopedic appliance. (33) The distribution of patients receiving treatment with functional orthopedic appliance were: JIA $0-0(n=13 / 17,77 \%)$, JIA $0-A(n=18 / 20,90 \%)$, and JIA $0-B(n=10 / 10,100 \%)$. No significant intergroup difference was observed in the duration of treatment with functional orthopedic appliance at the time of CBCT.

Control subjects: Nineteen subjects were included. Based on their TMJ abnormality scores, the control group comprised the following subgroups: 0-0 $(n=14), 0-B(n=3), A-A(n=1)$, and B-B $(n=1)$, which indicates that abnormal joint appearances were also present among the control subjects as a random finding. The mean age at CBCT for the control subjects was 13.2 years (std. 1.6) (Table 2).

The intra-rater reliability of the radiological TMJ abnormality scorings revealed an agreement of 83.3 percent $(\kappa=0.67)$ in the assessment of normal versus abnormal radiological TMJ appearance. In the TMJ assessment of joints that were scored with "abnormal findings," an intra-rater agreement of $76.7 \%(\kappa=0.63)$ was found in the subgroups (score A versus score $B$ ). According to Fleiss et al., this represents a fair agreement $(\kappa=0.4<$ fair agreement $\leq 0.75) .{ }^{34}$

\section{Dentofacial asymmetry}

Inter-group differences in dentofacial asymmetry are listed in Table 3. The primary ANOVA tests revealed significant inter-group differences in the following outcome variables $(p<0.006)$ : 1) mandibular posterior height, 2) condylar height, 3) axial plane to gonion, 4) mandibular 
transversal width at the gonion level, and 5) gonion plane angle (Table 3). The results of the secondary post-ANOVA $t$-tests (level of significance $\mathrm{p}<0.008$ ) are shown in Fig. 4 . The significant results are presented below.

Condylar height: A significantly larger degree of asymmetry was observed in the JIA 0-A and 0-B groups than in the control group. Additionally, the condylar height asymmetry was significantly more pronounced in JIA 0-A patients than in the JIA 0-0 group. No significant differences were observed between the control and JIA 0-0 groups or between the JIA 0-A and JIA 0-B groups (Table 3 and Fig. 4a).

Mandibular posterior height: Significantly larger asymmetries were found in the JIA 0-A and JIA 0-B groups than in the control and JIA 0-0 groups. No significant differences were observed between the control group and the JIA 0-0 group or between the JIA 0-A and JIA 0B groups (Table 3 and Fig. 4b).

Axial plane to gonion: Asymmetry was significantly larger in the JIA 0-A and 0-B groups than in the control group. Significant differences were not found between the three JIA groups or between the JIA 0-0 and control groups (Table 3 and Fig. 4d).

Transversal width at gonion level: A significant difference was observed between the JIA 0-0 group and JIA 0-B group. However, although significant, this inter-group difference in ratio did not exceed the smallest detectable difference of this specific outcome variable (Table 3 and Fig. 4h).

Gonion plane angle: A smaller angle was observed in the JIA 0-A and 0-B groups than in the control group, indicating a larger difference in the vertical position of the gonion point right and left. JIA 0-0 was not significantly different from the two other groups, JIA 0-A and JIA 0B (Table 3 and Fig. 4i). The outcome variables with no significant differences were ramus height, axial plane to inferior molar, mandibular length and jaw angle (Table 3 and Fig. 4c, e, $\mathrm{f}, \mathrm{g})$.

The intra-rater reliability of the nine outcome variables showed acceptable correlation levels between the duplicate assessments ( $r>0.90)$ with the exception of the outcome measure reflecting the transversal width at the gonion level $(r=0.69)$. The ranges of the smallest detectable differences for the inter-side ratios were calculated for each of the nine outcome variables using Bland-Altman plots (range=0.02-0.09). The smallest detectable differences for the two angular outcome variables were 0.02 degrees for the "jaw angle" and 1.32 degrees for the "gonion plane angle" outcome measure. 


\section{Discussion}

Dentofacial asymmetry is a common feature in patients with JIA and unilateral TMJ arthritis involvement. ${ }^{24,26,29}$ Minor dentofacial and mandibular asymmetries are also well known findings in a normal population; however, a precisely defined normal range has not been established. ${ }^{35}$ In the present study, the JIA patients with unilateral TMJ abnormalities exhibited significantly more severe dentofacial inter-side asymmetries than the JIA patients without TMJ abnormalities and control subjects. This is in accordance with other studies reporting pronounced asymmetries and smaller mandibular dimensions in JIA patients with condylar abnormalities. ${ }^{14,18,24}$ Koos et al. investigated mandibular asymmetries in 23 JIA patients using CBCT and compared them with 23 age- and gender-matched controls. ${ }^{29}$ Significantly more pronounced asymmetries were found in the patients with JIA than in the controls. However, Koos et al. made no attempt to correlate the intra-individual asymmetries with TMJ abnormalities, as was done in this study. Huntjens et al. examined the degree of condylar head asymmetry in children affected with JIA based on CBCT. ${ }^{26}$ Their results showed that asymmetry of the condyles was frequently present; however, condylar asymmetry did not correlate with facial 3D appearance.

To our knowledge, this is the first CBCT-based human study to investigate the association between types of radiological TMJ abnormalities and dentofacial development in JIA patients with TMJ involvement. In the present study, mandibular dysmorphic development was affected to the same extent in patients with either unilateral condylar deformations (score A) or unilateral erosions (score B). The cross-sectional nature of our study restricts our ability to draw conclusions regarding the causal relationship between TMJ abnormalities and dysmorphic dentofacial development; however, our findings call for a larger debate. It is not our impression that the two radiological features (deformation and erosion) necessarily represent a disease progression going from A towards B when attention is paid to general dentofacial development. Instead, A and B could be regarded as two expressions of the same process that vary over time based on the severity of the TMJ inflammation. This hypothesis is supported by a 5-year cohort follow-up study providing evidence for a condylar regenerative capacity in patients with low disease activity. ${ }^{9}$ We allow ourselves to hypothesize that TMJs with scores $\mathrm{A}$ and $\mathrm{B}$ are both expressions of a reaction to inflammation in the TMJs where category A is likely to represent a stable phase and category B represent a reaction to a more active high inflammatory phase. Future studies based on contrast-enhanced MRI techniques are needed to confirm this hypothesis. Notably, in light of mandibular development, it remains an open 
question whether the presence of erosion (score B) can be considered worse than the presence of deformation (score A) or vice versa. The overlap of deformation (Score A) and erosions (Score B) in $30 \%$ (9/30 joints) of the joints assigned to an abnormal radiological appearance leads to the question of whether it is useful to distinguish between TMJ deformations and erosions in future scoring system when the results provide little information on general dentofacial development. Future longitudinal studies are warranted to assess the association between the intra-articular conditions and dysmorphic dentofacial development. Additionally, there is a need to develop 3D CBCT-based cephalometric standards for the assessment of JIAspecific dentofacial morphological changes.

In a recent publication from Peck et al., condylar deformation (flattening) was considered an indeterminate finding and was not included in the diagnostic algorithm for TMJ arthritis caused by "systemic arthritides." ${ }^{36}$ In support of this viewpoint, 13\% (5/38) of the TMJs in the nonJIA control group in our study were assigned an "abnormal" condylar score. However, our findings do not support this viewpoint in terms of patients with JIA, and we suggest an alternative approach to JIA radiological evaluation: the presence of an abnormal radiological joint appearance should always call for further assessment of dentofacial morphology despite the type of condylar deformity revealed by radiological evaluation (deformation or erosion).

We found the site of the asymmetry to be primarily related to a short condyle on the affected side. Significant differences were found for mandibular posterior height in JIA 0-A and JIA 0B when compared to control and JIA 0-0 groups; however, there were no significant differences for ramus height, indicating that a shorter condyle was responsible for the decreased posterior face height in our patient group. No further morphological differences were found. The reason for a lack of other deformities, such as inter-side difference in Ramus height and occlusal canting (inter-side difference in axial plane to Minf), could be ascribed to the orthopedic treatment that 41 of the patients received after a diagnosis of TMJ arthritis had been confirmed.

To our knowledge, this is the largest CBCT-based case-control study of its kind on JIA. However, there are limitations to the present study. First, only a fair agreement $(\kappa=0.67)$ was obtained in the radiological assessment of intra-rater reliability between normal and abnormal TMJ appearance. This may be explained by the fact that the subchondral cortical bone is under development in children and adolescents, which may hamper radiological assessments in the age group included in this study. ${ }^{37}$ The kappa value for differentiating the abnormal morphology into group A or B was slightly lower $(\kappa=0.63)$, which may be explained by the 
fact that some joints exhibited features from both groups that also may have interfered with interpretation. Second, a correlation between the duration of TMJ arthritis and the degree of facial asymmetry was not considered because initial MRI verification of TMJ arthritis was not routinely conducted in all patients. Third, the included JIA patients represented a group of well treated patients with orthopedic splints; this may have moderated the intra-patient asymmetries. To avoid this bias, the study should have been conducted with untreated JIA patients; however, this would have been unethical in our opinion. Forty-one of the 47 included JIA patients were undergoing or had previously received functional appliance treatment with an orthopedic distraction splint. In five of the remaining six patients, an orthopedic distraction splint was fabricated immediately after the CBCT scan date and functional treatment was commenced, whereas the final patient was followed to observe further development. There were no differences in treatment protocols offered to the three JIA groups. We previously showed that treatment with an orthopedic distraction splint facilitates comparable inter-side growth rates in patients with unilateral TMJ arthritis and prevents mandibular asymmetry from being exacerbated. ${ }^{33}$ All patients received routine orofacial examination, and distraction splint treatment was initiated as soon as facial asymmetry was noted; thus, we hypothesize that only a minor aggravation of asymmetries occurred after the initiation of the distraction splint treatment.

\section{Conclusions}

- JIA patients with unilateral condylar abnormalities (deformation or erosion) exhibited significantly more severe dentofacial asymmetries than JIA patients without condylar abnormalities and control subjects.

- A similar degree of dentofacial asymmetry was observed in the two groups of patients with unilateral condylar abnormalities (deformation or erosion).

- Mandibular asymmetry was exclusively related to a short condyle on the affected side.

- In patients with JIA, both deformation and erosion were present in $30 \%$ of the TMJs examined.

- Radiological signs of condylar abnormalities (deformation and/or erosion) were observed in $13 \%$ of the TMJs in the non-JIA control group.

- Generally, in JIA, the presence of abnormal radiological TMJ appearance should always call for the further assessment of dentofacial morphology. 


\section{Acknowledgement}

We thank Professor Tore A. Larheim for his help and guidance during the radiological assessments.

\section{Funding}

No funding was received for this project.

\section{References}

1. Larheim TA, Doria AS, Kirkhus E, Parra DA, Kellenberger CJ, Arvidsson LZ. TMJ imaging in JIA patients; An overview. Semin Orthod.2015; 21:102-10.

2. Arvidsson LZ, Fjeld MG, Smith HJ, Flato B, Ogaard B, Larheim TA. Craniofacial growth disturbance is related to temporomandibular joint abnormality in patients with juvenile idiopathic arthritis, but normal facial profile was also found at the 27 -year follow-up. Scand J Rheumatol. 2010;39:373-9.

3. Arvidsson LZ, Flato B, Larheim TA. Radiographic TMJ abnormalities in patients with juvenile idiopathic arthritis followed for 27 years. Oral Surg Oral Med Oral Pathol Oral Radiol Endod. 2009;108:114-23.

4. Bakke M, Zak M, Jensen BL, Pedersen FK, Kreiborg S. Orofacial pain, jaw function, and temporomandibular disorders in women with a history of juvenile chronic arthritis or persistent juvenile chronic arthritis. Oral Surg Oral Med Oral Pathol Oral Radiol Endod. 2001;92:406-14.

5. Engstrom AL, Wanman A, Johansson A, Keshishian P, Forsberg M. Juvenile arthritis and development of symptoms of temporomandibular disorders: a 15-year prospective cohort study. J Orofac Pain. 2007;21:120-6.

6. Fjeld MG, Arvidsson LZ, Stabrun AE, Birkeland K, Larheim TA, Ogaard B. Average craniofacial development from 6 to 35 years of age in a mixed group of patients with juvenile idiopathic arthritis. Acta Odontol Scand. 2009;67:153-60.

7. Kuseler A, Pedersen TK, Gelineck J, Herlin T. A 2 year followup study of enhanced magnetic resonance imaging and clinical examination of the temporomandibular joint in children with juvenile idiopathic arthritis. J Rheumatol. 2005;32:162-9.

8. Stoustrup P, Kristensen KD, Verna C, Kuseler A, Herlin T, Pedersen TK. Orofacial symptoms related to temporomandibular joint arthritis in juvenile idiopathic arthritis: smallest detectable difference in self-reported pain intensity. J Rheumatol. 2012;39:2352-8.

9. Twilt M, Schulten AJ, Verschure F, Wisse L, Prahl-Andersen B, Suijlekom-Smit LW. Long-term followup of temporomandibular joint involvement in juvenile idiopathic arthritis. Arthritis Rheum. 2008;59:546-52.

10. Ahmed N, Petersson A, Catrina Al, Mustafa H, Alstergren P. Tumor necrosis factor mediates temporomandibular joint bone tissue resorption in rheumatoid arthritis. Acta Odontol Scand. 2015;73:232-40.

11. Billiau AD, Hu Y, Verdonck A, Carels C, Wouters C. Temporomandibular Joint Arthritis in Juvenile Idiopathic Arthritis: Prevalence, Clinical and Radiological Signs, and Relation to Dentofacial Morphology. J Rheumatol. 2007;34:1925-33.

12. Hu YS, Schneiderman ED, Harper RP. The temporomandibular joint in juvenile rheumatoid arthritis: Part II. Relationship between computed tomographic and clinical findings. Pediatr Dent. 1996;18:312-9.

13. Kjellberg H. Juvenile chronic arthritis. Dentofacial morphology, growth, mandibular function and orthodontic treatment. Swed Dent J Suppl. 1995;109:1-56. 
14. Kjellberg H. Craniofacial growth in juvenile chronic arthritis. Acta Odontol Scand. 1998;56:360-5.

15. Kirkhus E, Arvidsson LZ, Smith HJ, Flato B, Hetlevik SO, Larheim TA. Disk abnormality coexists with any degree of synovial and osseous abnormality in the temporomandibular joints of children with juvenile idiopathic arthritis. Pediatr Radiol 2016;46:331-41.

16. Kristensen KD, Hauge EM, Dalstra M, Stoustrup P, Kuseler A, Pedersen TK, et al. Association between condylar morphology and changes in bony microstructure and sub-synovial inflammation in experimental temporomandibular joint arthritis. J Oral Pathol Med. 2011;40:111-20. 17. Stoustrup P, Kristensen KD, Kuseler A, Gelineck J, Cattaneo PM, Pedersen TK, et al. Condylar lesions in relation to mandibular growth in untreated and intra-articular corticosteroidtreated experimental temporomandibular joint arthritis. Clin Exp Rheumatol. 2010;28:576-83.

18. Twilt M, Schulten AJ, Nicolaas P, Dulger A, Suijlekom-Smit LW. Facioskeletal changes in children with juvenile idiopathic arthritis. Ann Rheum Dis. 2006;65:823-5.

19. Peltomäki T, Kreiborg S, Pedersen, TK; Ogaard, B. Craniofacial growth and dentoalveolar development in juvenile idiopathic arthritis patients. Semin Orthod. 2015;21:84-93. 20. Koos B, Tzaribachev N, Bott S, Ciesielski R, Godt A. Classification of temporomandibular joint erosion, arthritis, and inflammation in patients with juvenile idiopathic arthritis. J Orofac Orthop. 2013;74:506-19.

21. Vaid YN, Dunnavant FD, Royal SA, Beukelman T, Stoll ML, Cron RQ. Imaging of the temporomandibular joint in juvenile idiopathic arthritis. Arthritis Care Res (Hoboken). 2014;66:47-54. 22. Kristensen KD, Stoustrup P, Kuseler A, Pedersen TK, Nyengaard JR, Hauge EM, et al. Quantitative histological changes of repeated antigen-induced arthritis in the temporomandibular joints of rabbits treated with intra-articular corticosteroid. J Oral Pathol Med. 2008;37:437-44.

23. Ronchezel MV, Hilario MO, Goldenberg J, Lederman HM, Faltin K, Jr., de Azevedo MF, et al. Temporomandibular joint and mandibular growth alterations in patients with juvenile rheumatoid arthritis. J Rheumatol. 1995;22:1956-61.

24. Stabrun AE, Larheim TA, Hoyeraal HM, Rosler M. Reduced mandibular dimensions and asymmetry in juvenile rheumatoid arthritis. Pathogenetic factors. Arthritis Rheum. 1988;31:602-11. 25. Arvidsson LZ, Smith HJ, Flato B, Larheim TA. Temporomandibular joint findings in adults with long-standing juvenile idiopathic arthritis: $\mathrm{CT}$ and MR imaging assessment. Radiology. 2010;256:191-200.

26. Huntjens E, Kiss G, Wouters C, Carels C. Condylar asymmetry in children with juvenile idiopathic arthritis assessed by cone-beam computed tomography. Eur J Orthod. 2008;30:545-51.

27. Farronato G, Garagiola U, Carletti V, Cressoni P, Mercatali L, Farronato D. Change in condylar and mandibular morphology in juvenile idiopathic arthritis: Cone Beam volumetric imaging. Minerva Stomatol. 2010;59:519-34.

28. Lima Ferraz Junior AM, Devito KL, Guimaraes JP. Temporomandibular disorder in patients with juvenile idiopathic arthritis: clinical evaluation and correlation with the findings of cone beam computed tomography. Oral Surg Oral Med Oral Pathol Oral Radiol Endod.. 2012;114:E51-57.

29. Koos B, Gassling V, Bott S, Tzaribachev N, Godt A. Pathological changes in the TMJ and the length of the ramus in patients with confirmed juvenile idiopathic arthritis. J Craniomaxillofac Surg 2014;42:1802-7.

30. Larheim TA, Abrahamsson AK, Kristensen M, Arvidsson LZ. Temporomandibular joint diagnostics using cone beam computed tomography. Dentomaxillofac Radiol. 2014:20140235.

31. Petty RE, Southwood TR, Baum J, Bhettay E, Glass DN, Manners P, et al. Revision of the proposed classification criteria for juvenile idiopathic arthritis: Durban, 1997. J Rheumatol. 1998;25:1991-4.

32. Bland JM, Altman DG. Statistical methods for assessing agreement between two methods of clinical measurement. Lancet. 1986;1:307-10. 
33. Stoustrup P, Kuseler A, Kristensen KD, Herlin T, Pedersen TK. Orthopaedic splint treatment can reduce mandibular asymmetry caused by unilateral temporomandibular involvement in juvenile idiopathic arthritis. Eur J Orthod. 2013;35:191-8

34. Fleiss J LLB, A; Paik M,C. Statistical methods for rates and proportions. J.Wiley, Hoboken, N.J); 2003.

35. Liukkonen M, Sillanmaki L, Peltomaki T. Mandibular asymmetry in healthy children. Acta Odontol Scand. 2005;63:168-72.

36. Peck CC, Goulet JP, Lobbezoo F, Schiffman EL, Alstergren P, Anderson GC, et al. Expanding the taxonomy of the diagnostic criteria for temporomandibular disorders. J Oral Rehabil 2014;41:2-23.

37. Lei J, Liu MQ, Yap AU, Fu KY. Condylar subchondral formation of cortical bone in adolescents and young adults. Br J Oral Maxillofac Surg. 2013;51:63-8. 
Table 1. Definition and description of the landmarks, planes and variables used in the study. Colors refer to the planes illustrated in Fig. 2.

\begin{tabular}{|c|c|c|}
\hline Landmarks & Definition & Abbreviation \\
\hline Condyle point & The midpoint on the superior surface of the condyle & Cor/Col \\
\hline Gnathion & The lowest point on the lower border of the chin & $\mathrm{Gn}$ \\
\hline Gonion (right/left) & $\begin{array}{l}\text { Constructed by the bisection by the angle formed by the tangents } \\
\text { to the lower and posterior borders of the mandible }\end{array}$ & Gor/Gol \\
\hline $\begin{array}{l}\text { Incisura point } \\
\text { (right/left) }\end{array}$ & $\begin{array}{l}\text { Lowest point in the concavity between processus coronoideus } \\
\text { and processus condylaris in relation to the Axial Plane }\end{array}$ & Incr/Incl \\
\hline $\begin{array}{l}\text { Latero-orbital point } \\
\text { (right/left) }\end{array}$ & $\begin{array}{l}\text { At the zygomaticofrontal suture at the lateral aspect of the orbit } \\
\text { wall }\end{array}$ & LOr/LO1 \\
\hline $\begin{array}{l}\text { Lower first molar point } \\
\text { (right/left) }\end{array}$ & The mesio-buccal cusp of the lower first molar & Minfr/Minfl \\
\hline Nasion & Midpoint between maxillary-nasal-frontal right and left junction & $\mathrm{N}$ \\
\hline Sella turcica & The centre of the hypophyseal fossa & $\mathrm{S}$ \\
\hline Planes & Definition & Color \\
\hline Axial plane & Through S, LOr and LOl & Blue \\
\hline Mid-sagittal plane & Through N, S and perpendicular to the axial plane & Dark blue \\
\hline Coronal plane & $\begin{array}{l}\text { Through } \mathrm{S} \text { and perpendicular to axial plane and mid-sagittal } \\
\text { plane }\end{array}$ & Pink \\
\hline Inc plane (right/left) & $\begin{array}{l}\text { Through Inc (left / right) and perpendicular to the mid-sagittal } \\
\text { plane and the coronal plane. One plane for each side was created }\end{array}$ & Purple \\
\hline Gonion plane & Plane through Gonion (right/left) and Gnathion & \\
\hline Variables & Definition & \\
\hline $\begin{array}{l}\text { Mandibular posterior } \\
\text { height (right/left) }\end{array}$ & From Go to Co (mm) & \\
\hline $\begin{array}{l}\text { Condylar height } \\
\text { (right/left) }\end{array}$ & From Co to Inc plane & \\
\hline $\begin{array}{l}\text { Ramus height } \\
\text { (right/left) }\end{array}$ & Go to Inc plane (mm) & \\
\hline
\end{tabular}


Axial plane-Go

(right/left)

Axial plane-Minf (right/left)

Mandibular length

right/left)

Jaw angle (right/left )

Transversal width at gonion level (right/left)

Gonion plane angle
Axial plane to gonion (mm)

Axial plane to lower molar mesio-buccal cusp tip (mm)

Gn to Go (mm)

Angle between the line connecting $\mathrm{Co}$ and Go and the line connecting Go and Gn (degrees)

Distance from mid-sagittal plane to gonion (mm)

Angle between gonial plane and the mid-sagittal plane (degrees) measured to the affected side / group 0-0 to the smallest side 
Table 2. Characteristics of patients with JIA and control subjects included in the study.

CBCT: cone beam computed tomography; NSAIDs: non-steroidal anti-inflammatory drugs;

DMARDs: disease-modifying anti-rheumatic drugs.

\begin{tabular}{|c|c|c|c|c|}
\hline Cohort characteristics & Control group & JIA 0-0 & JIA 0-A & JIA 0-B \\
\hline Number & 19 & 17 & 20 & 10 \\
\hline Females, number & 12 & 11 & 14 & 7 \\
\hline Males, number & 7 & 6 & 6 & 3 \\
\hline $\begin{array}{l}\text { Mean age at } \mathrm{CBCT} \text {, years (sd } \\
\text { years) }\end{array}$ & $13.2(1.6)$ & $13.2(2.6)$ & $12.8(2.7)$ & $11.4(2.5)$ \\
\hline $\begin{array}{l}\text { Present or earlier functional } \\
\text { treatment, number }\end{array}$ & - & 13 & 18 & 10 \\
\hline \multicolumn{5}{|l|}{ JIA subcategories } \\
\hline Oligoarticular extended & & 3 & 5 & 2 \\
\hline Oligoarticular persistent & & 7 & 4 & 1 \\
\hline Polyarticular & & 3 & 10 & 4 \\
\hline Systemic & & 1 & - & - \\
\hline Psoriatic & & 1 & - & - \\
\hline Enthesitis related arthritis & & - & - & 1 \\
\hline Undifferentiated & & 2 & 1 & 2 \\
\hline \multicolumn{5}{|l|}{ Medication } \\
\hline No medication & & 5 & 8 & 1 \\
\hline NSAIDs & & 8 & 7 & 5 \\
\hline DMARDs & & 4 & 6 & 8 \\
\hline Biologics & & 5 & 3 & 6 \\
\hline Combination of 2 drugs & & 3 & 4 & 4 \\
\hline Combination of 3 drugs & & - & - & 3 \\
\hline \multicolumn{5}{|l|}{ Disease duration, years** } \\
\hline Mean years (sd) & & $7(3.4)$ & $5(3.7)$ & $6(4.4)$ \\
\hline$<1$ & & 1 & - & 1 \\
\hline $0-3$ & & 1 & 6 & 2 \\
\hline $3-5$ & & 5 & 5 & 3 \\
\hline
\end{tabular}




\begin{tabular}{llll}
\hline$>5$ & 10 & 8 & 4 \\
TMJ involvement, years*** & & & 4 \\
$<1$ & 4 & 3 & 1 \\
$0-2$ & 5 & 7 & 2 \\
$3-5$ & 3 & 2 & 3 \\
$>5$ & 5 & 8 & \\
\hline
\end{tabular}


Table 3. ANOVA tests analyzing ratios and angels of inter-side dentofacial asymmetries in patients and control subjects. Numbers indicate inter-side ratios, standard deviations in brackets. $/ *=$ linear (ratio) measurement, $/{ }^{\circ}=$ degrees, $* *=$ level of significance $p<0.006$. PostANOVA tests were only performed for outcome variables in which a statistically significant difference was observed in the primary ANOVA test. N.S.=non-significant.

\begin{tabular}{|c|c|c|c|c|c|}
\hline $\begin{array}{l}\text { Outcome } \\
\text { measures }\end{array}$ & Control group & $\begin{array}{l}\mathrm{JIA} \\
0-0\end{array}$ & $\begin{array}{l}\mathrm{JIA} \\
0-\mathrm{A}\end{array}$ & $\begin{array}{l}\text { JIA } \\
0-B\end{array}$ & $\begin{array}{l}\text { ANOVA } \\
\text { p-value** }\end{array}$ \\
\hline $\begin{array}{l}\text { Condylar height } \\
\qquad / *\end{array}$ & $0.95(0.05)$ & $0.92(0.07)$ & $0.78(0.12)$ & $0.80(0.17)$ & $<0.001$ \\
\hline $\begin{array}{l}\text { Mandibular } \\
\text { posterior } \\
\text { height/* }\end{array}$ & $0.98(0.02)$ & $0.96(0.03)$ & $0.88(0.06)$ & $0.90(0.07)$ & $<0.001$ \\
\hline Ramus height/* & $0.98(0.03)$ & $0.96(0.03)$ & $0.94(0.07)$ & $0.95(0.06)$ & N.S. \\
\hline $\begin{array}{l}\text { Axial plane to } \\
\text { gonion } \text { / }^{*}\end{array}$ & $0.99(0.01)$ & $0.98(0.02)$ & $0.95(0.04)$ & $0.95(0.04)$ & $<0.001$ \\
\hline $\begin{array}{l}\text { Axial plane to } \\
\text { molar inf. /* }\end{array}$ & $0.99(0.01)$ & $0.98(0.02)$ & $0.97(0.03)$ & $0.98(0.03)$ & N.S. \\
\hline $\begin{array}{l}\text { Mandibular } \\
\text { length /* }\end{array}$ & $0.99(0.01)$ & $0.98(0.01)$ & $0.98(0.03)$ & $0.98(0.04)$ & N.S \\
\hline Jaw angle /० & $0.98(0.02)$ & $0.99(0.01)$ & $1.00(0.03)$ & $0.99(0.01)$ & N.S. \\
\hline $\begin{array}{c}\text { Transversal } \\
\text { width at gonion } \\
\text { level/* }\end{array}$ & $0.97(0.03)$ & $0.96(0.04)$ & $1.04(0.08)$ & $1.0(0.04)$ & $<0.001$ \\
\hline $\begin{array}{c}\text { Gonion plane } \\
\text { angle / }\end{array}$ & $89.6(0.60)$ & $88.9(0.98)$ & $88.1(1.98)$ & $87.9(1.48)$ & 0.002 \\
\hline
\end{tabular}



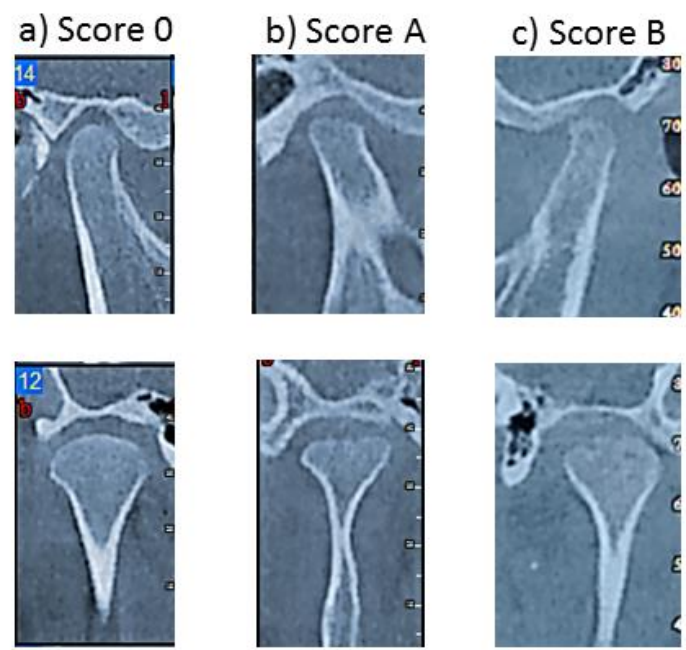

a) Score 0

b) Score $A$

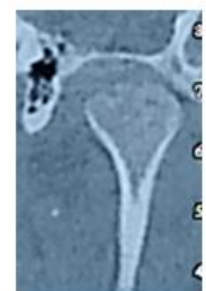

c) Score B

Figure 1. Scoring of condylar morphology based on radiological TMJ appearance. a) Condylar score 0: Normal, normal shape with smooth and intact outline/surface. b) Condylar score A: Deformed, marked flattening or other changes in shape with smooth and intact outline/surface. c) Condylar score B: Erosive, disruption of outline or uneven surface due to cyst or erosion. 

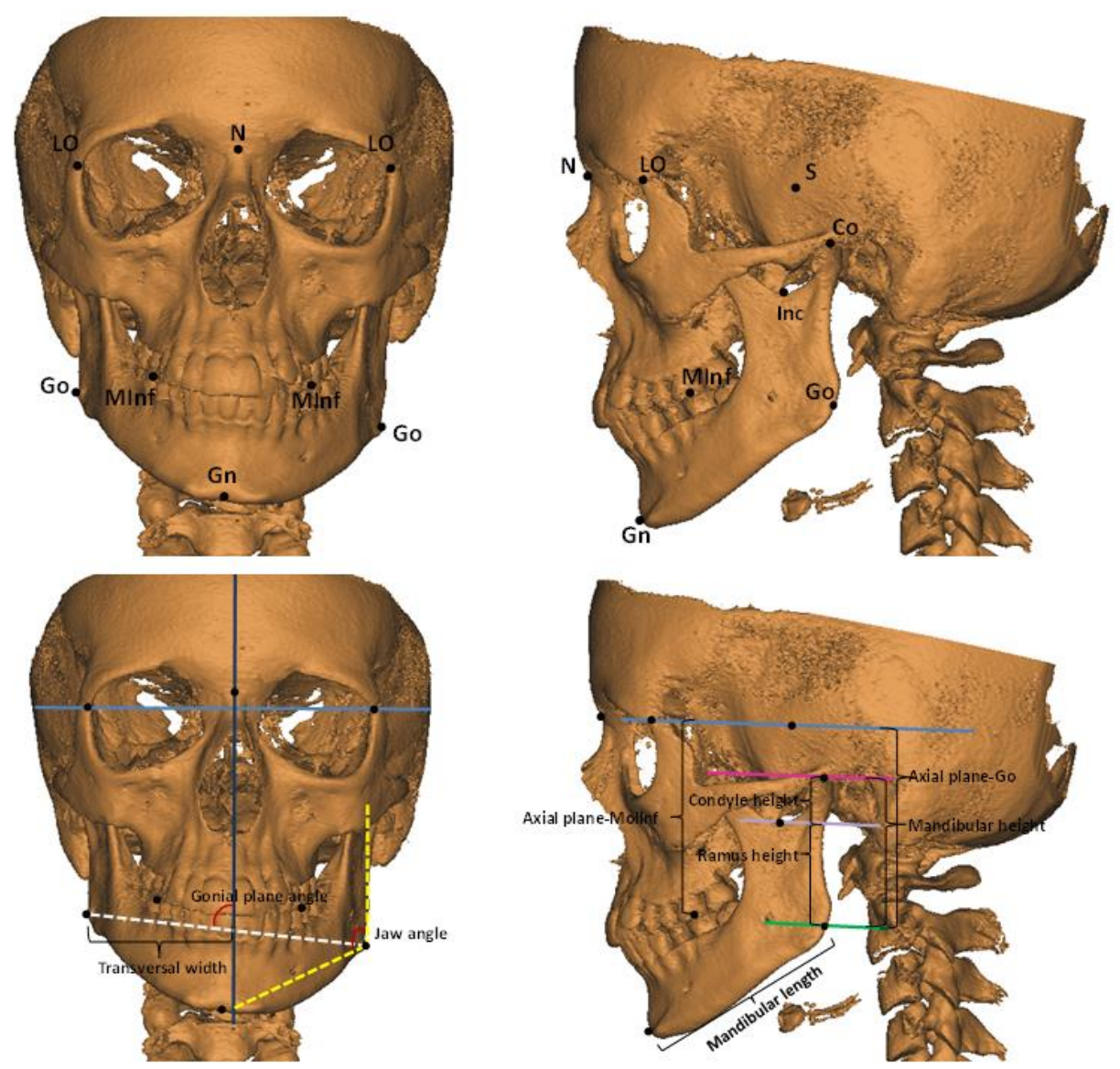

Figure 2. Illustration of the landmarks, planes and variables from the $3 \mathrm{D}$ analysis. a $\& \mathrm{~b}$ : Frontal and sagittal view with landmarks. c \& d: Frontal and sagittal view with planes and variables. Color code of planes: axial plane, blue; midsagittal plane, dark blue; coronal plane, pink; incisura plane (left / right), purple. Further descriptions of landmarks and variables are presented in Table 1. 


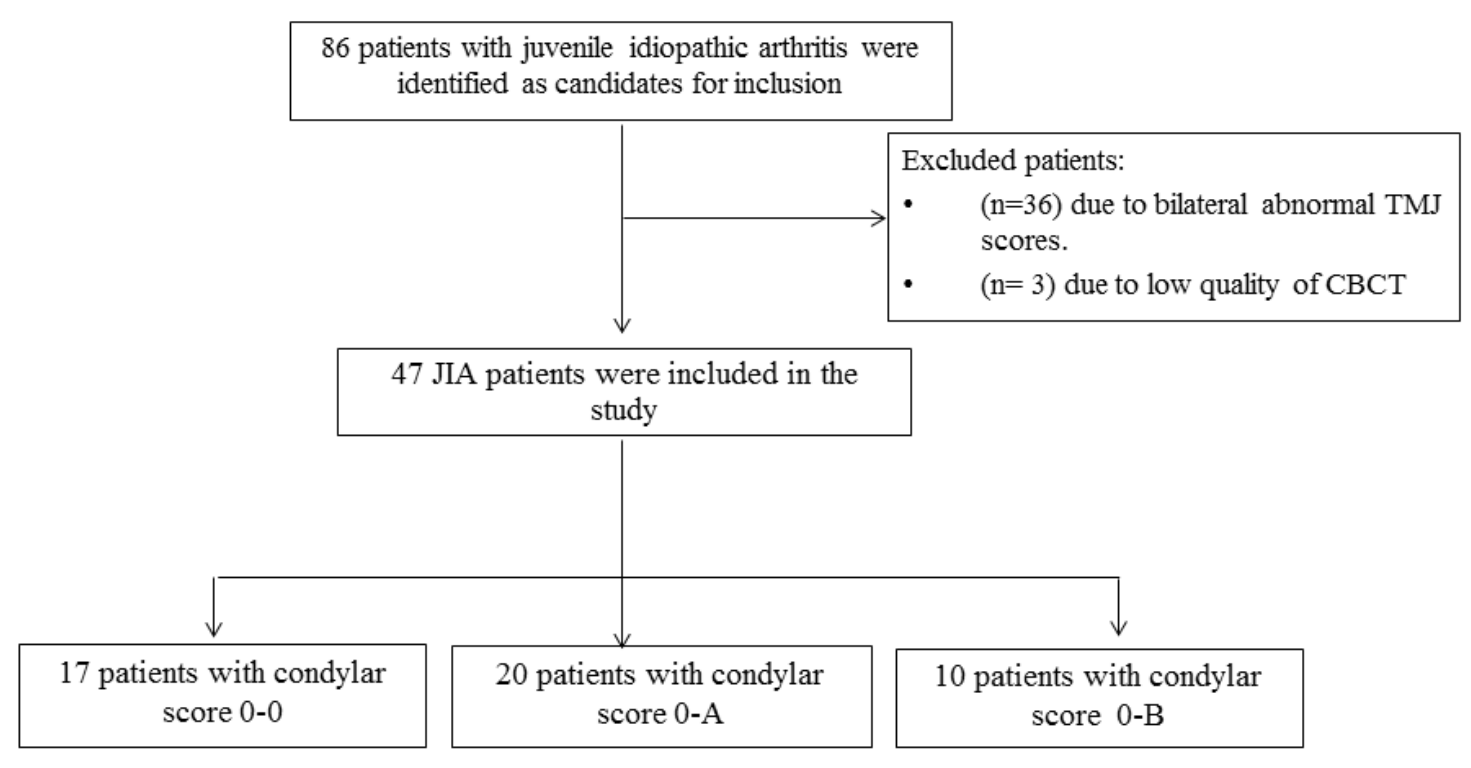

Figure 3. Flowcharts of included JIA patients. 

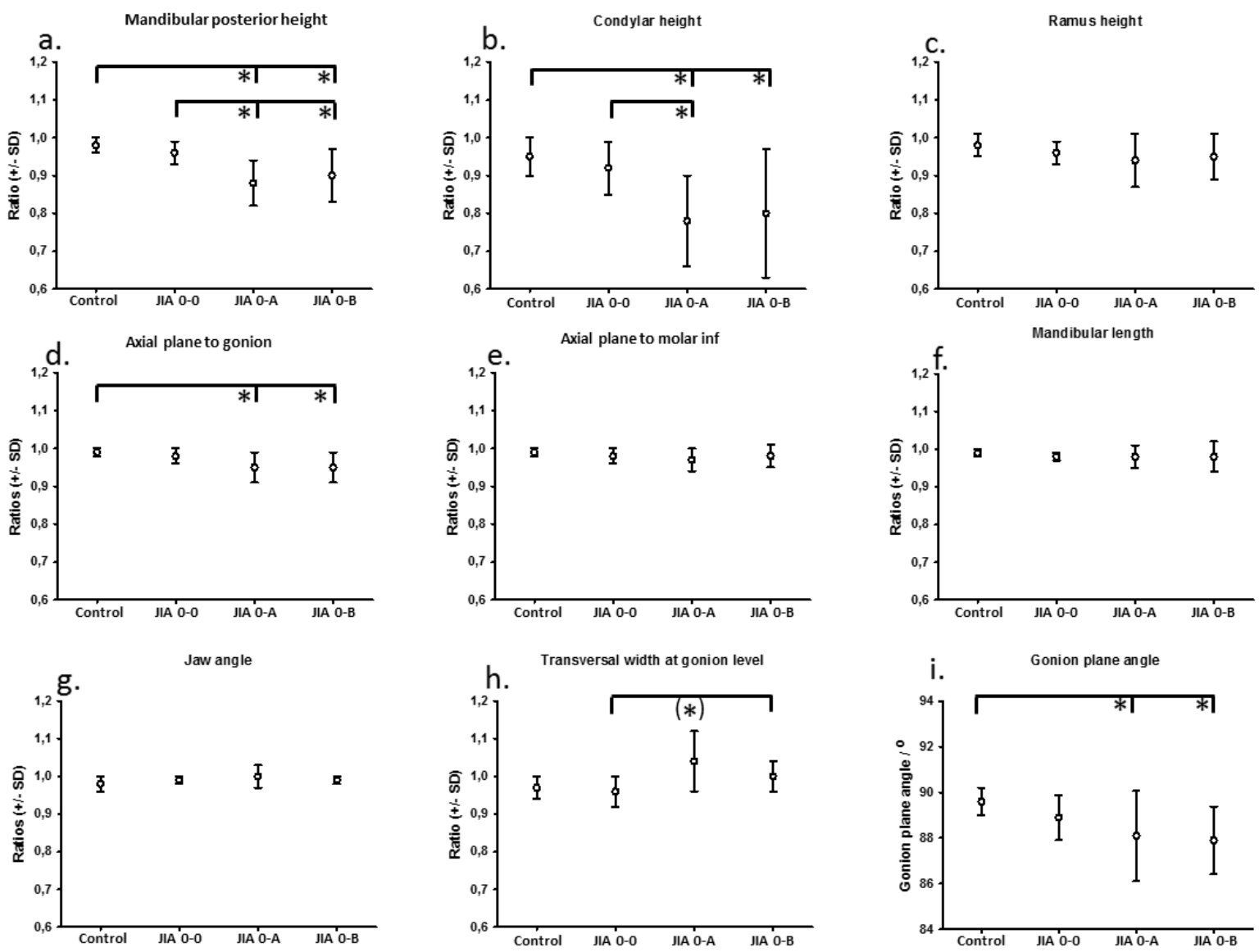

Figure 4. Evaluation of inter-side differences. The results of post-ANOVA $t$-tests illustrated graphically for the nine outcome variables. A ratio of 1 indicates perfect symmetry, whereas a reduced ratio indicates dentofacial asymmetry between the two sides. The ratio decreases with increased dentofacial asymmetry. In the angular measurement, an angle of 90 degrees indicates symmetry, whereas an angle below 90 degrees indicates increased asymmetry. A smaller angle indicates more asymmetry between the two sides. * ${ }^{*}$ level of significance $\mathrm{p}<0.008$. $(*)=$ significant but not exceeding the smallest detectable difference. 